\title{
ESTUDO DA ESTABILIDADE DE ANTOCIANINAS EXTRAÍDAS DOS FRUTOS DE AÇAÍ (Euterpea oleracea Mart.)
}

\author{
Z. G. $\operatorname{COSTA}^{1}$, C. de L. O. C. e SILVA², C. M. L. $\operatorname{COSTA}^{3}$ e L. J. G. FARIA ${ }^{3}$
}

${ }^{1}$ Bolsista de Iniciação Científica- PIBIC, FEQ/ UFPA

${ }^{2}$ Mestranda do Programa de Pós Graduação em Engenharia Química- PPGEQ/ UFPA

${ }^{3,3}$ Docente da Faculdade de Engenharia Química- FEQ/UFPA

E-mail para contato: cmlc@ufpa.br

\begin{abstract}
RESUMO - O açaí, como é conhecido o fruto do açaizeiro (Euterpe oleracea Mart.), produz uma bebida de grande valor nutricional e consumida em todo o mundo. Porém, o fruto apresenta também aplicação industrial como corante natural a partir da extração de antocianinas responsáveis pela sua forte coloração. Tendo-se em vista a crescente demanda da indústria por corantes de origem natural em detrimento àqueles sintetizados artificialmente, estuda-se a estabilidade do extrato antociânico quando exposto à degradação luminosa com base em dados experimentais obtidos em câmaras contendo lâmpadas de LED com três diferentes potências: 4,5 e $10 \mathrm{~W}$. Os parâmetros utilizados para avaliar a estabilidade dos extratos do corante foram: concentração de antocianinas totais e parâmetros colorimétricos.
\end{abstract}

Palavras-chave: corante natural, açaí, estabilidade.

\section{INTRODUÇÃO}

O açaizeiro (Euterpe oleracea Mart.) é uma Arecaceae típica da região norte do Brasil, cujos frutos são caracteristicamente pequenos, arredondados e de coloração roxo-escuro (SOUSA et al, 2006). Foi observado nos últimos anos um crescimento de demanda no mercado nacional pelos derivados do fruto de açaí, despertando grande interesse em investimentos e pesquisas sobre o assunto. Este aumento pode ser atribuído às propriedades nutricionais do açaí, e por possuir um elevado teor de pigmentos chamados antocianinas, que, dentre outras características, são benéficos à saúde (ALEXANDRE, CUNHA e HUBINGER, 2004). Estes pigmentos são considerados instáveis a diversos fatores dentre os quais se destacam a luz e a temperatura de congelamento.

As cores são adicionadas em determinados produtos, principalmente para restituir a aparência original (afetada durante as etapas de processamento, estocagem, embalagem e distribuição) e para torná-lo visivelmente atraente (ajudando a identificar o aroma normalmente associado a determinados produtos. Os corantes advindos do açaí têm muitas vantagens, porém a principal desvantagem está no elevado custo de produção, e na baixa estabilidade a luz, temperatura, $\mathrm{pH}$ e presença de oxigênio quando comparados aos corantes artificiais. 

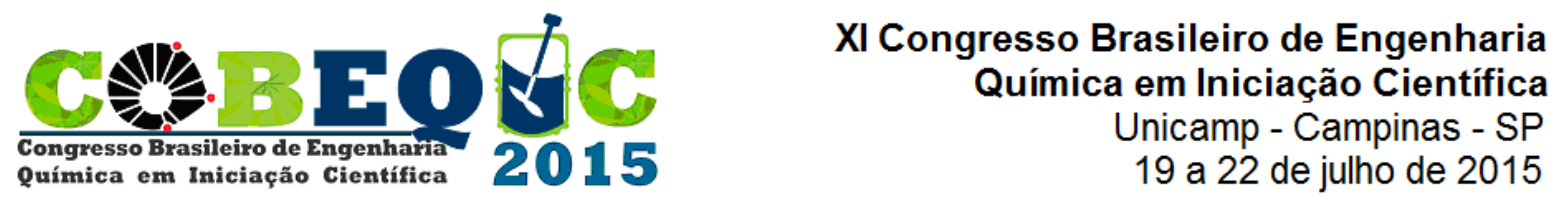

Desta forma, este trabalho visa estudar o efeito da luz sobre a deterioração de corantes contidos nos frutos de açaí, além de verificar a ordem de reação da degradação de antocianina sujeita a incidência de diferentes tipos de luz e propor modelos para a taxa de fotodegradação e degradação térmica com o tempo, em função do tipo de luz empregada e temperatura.

\section{MÉTODO}

O trabalho foi conduzido no Laboratório de Corantes (LabCor), locado na FEQ/UFPA, utilizando frutos inteiros e frescos de açaí proveniente do município do Acará, Estado do Pará. Os frutos foram imediatamente submetidos ao procedimento de extração do extrato antociânico.

Os extratos antociânicos foram obtidos segundo metodologia descrita por Constant (2003). Macerou-se o fruto em etanol 70\% e em pH 2,0 durante 48 horas ao abrigo da luz, a uma temperatura em torno de $30^{\circ} \mathrm{C}$, realizando posterior filtração em papel Whatman 1 .

O extrato foi distribuído em porções de $10 \mathrm{~mL}$ (porção de extrato utilizada na análise de teor de antocianinas totais) em tubos de ensaio vedados com parafilme e divididos em 3 amostras, estas colocadas em diferentes câmaras de fotodegradação (Figura 1) contendo lâmpadas de LED com potências de $4 \mathrm{~W}, 5 \mathrm{~W}$ e $10 \mathrm{~W}$. Estas formas de armazenamento tiveram como objetivo verificar as degradações dos compostos bioativos durante um período de observação de 30 dias, com análises realizadas em intervalos de tempo de 48 horas.

Figura 1 - Câmara de Fotodegradação.

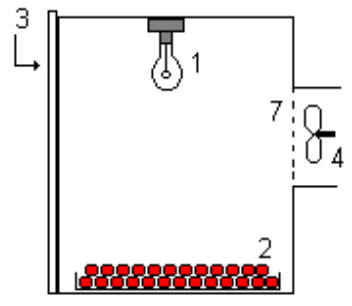

a) Vista Lateral

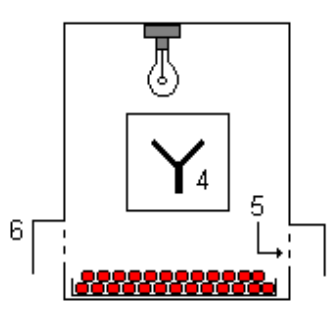

b) Vista Frontal

Onde: 1- Fonte Luminosa; 2- Recipiente com as amostras; 3- Abertura Frontal; 4- Sistema de exaustão; 5- Orifícios de saída de umidade; 6- Calhas para proteção de luminosidade externa e 7- Tela de plástico (escura).

As perdas de cor foram quantificadas por espectrofotometria. As análises quantitativas das antocianinas extraídas do fruto do açaí foram realizadas em triplicatas num espectrofotômetro UV- Visível, SHIMADZU modelo 160-A, com leituras de absorbâncias em comprimento de onda de $535 \mathrm{~nm}$.

A Tabela 1 sumariza os três modelos de ordem da reação utilizados na predição dos dados de degradação do pigmento do açaí. 
Tabela 1. Modelos utilizados na predição dos dados de degradação do pigmento.

\begin{tabular}{ccc}
\hline Ordem da reação & Modelos & Referência \\
\hline Ordem Zero & $C=C_{o}-k t$ & FU; LABUZA (1997) \\
Primeira Ordem & $C=C_{o} \exp ^{-k t}$ & FU; LABUZA (1997) \\
Segunda Ordem & $C=\frac{1}{k t+\frac{1}{C_{o}}}$ & FU; LABUZA (1997) \\
\hline
\end{tabular}

$\mathrm{C}=$ Concentração final; $\mathrm{C} 0=$ Concentração inicial; $\mathrm{k}=$ Constante de velocidade; $\mathrm{t}=$ tempo

A estimativa dos tempos de meia-vida para as diferentes ordens de reação cinética foi conduzida de acordo com as equações descritas na Tabela 2.

Tabela 2 - Tempos de meia-vida para as diferentes ordens de reação.

\begin{tabular}{ccc}
\hline Ordem aparente da reação & Tempo de meia vida $\left(\mathrm{t}^{1 / 2}\right)$ & Referência \\
\hline Ordem Zero & $t_{1 / 2}=\frac{C_{o}}{2 k}$ & TAOUKIS; LABUZA; \\
& $t_{1 / 2}=\frac{\ln 2}{k}$ & SAGUY (1997) \\
Primeira Ordem & $t_{1 / 2}=\frac{1}{k C_{o}}$ & TAOUKIS; LABUZA; \\
Segunda Ordem & SAGUY (1997) \\
\end{tabular}

$\mathrm{C}_{0}=$ Concentração inicial $; \mathrm{k}=$ Constante de velocidade $; \mathrm{t}=$ tempo

As análises das antocianinas extraídas dos frutos de açaí foram realizadas de acordo com o método de Fuleki e Francis (1968) posteriormente revisada por Lees e Francis (1972). Utilizando-se $\mathrm{pH}$ único, a cada 48 horas, alíquotas de $1 \mathrm{~mL}$ de extrato, exposto às diferentes luminosidades, foram diluídas em $10 \mathrm{~mL}$ de etanol $95 \%$ com $\mathrm{HCl} 1,5 \mathrm{~N}$ (85:15), homogeneizadas e levadas ao espectrofotômetro. Realizadas as leituras em triplicatas. Para a obtenção do cálculo da concentração foi utilizada a equação 1, a seguir:

$$
\text { Antocianinas totais }\left(\frac{m g}{100 g \text { de amostra }}\right)=\frac{A b s \lambda_{\text {máx }} \times F d \times 100}{E_{1 c m}^{1 \%}}
$$

Onde: $\mathrm{Abs}_{\lambda \text { máx }}$ é a absorbância no comprimento de onda máximo; Fd é o fator de diluição e $E_{1 c m}^{1 \%}$ é a absorvidade molar a $535 \mathrm{~nm}$ e corresponde a 98,29 em antocianinas de Cramberries.

\section{RESULTADOS}

\subsection{Teor de Antocianinas}

As leituras de absorbâncias feitas através do espectrofotômetro e as correspondentes concentrações de antocianinas estão sumarizadas na tabela 3. 


\section{Congresso Brasileiro de Engenharia Química em Iniciação Científica Unicamp - Campinas - SP 19 a 22 de julho de 2015}

Tabela 3- Teores de antocianinas totais em função do tempo de exposição a diferentes potências de lâmpadas LED.

\begin{tabular}{cccc}
\hline \multirow{2}{*}{$\mathrm{t}(\mathrm{h})$} & \multicolumn{3}{c}{ Antocianinas Totais $(\mathrm{mg} / 100 \mathrm{~g}$ de amostra) } \\
\cline { 2 - 4 } & $10 \mathrm{~W}$ & $5 \mathrm{~W}$ & $4 \mathrm{~W}$ \\
\hline 0 & 8,995 & 8,999 & 8,999 \\
48 & 3,352 & 3,899 & 4,521 \\
96 & 2,928 & 2,924 & 4,679 \\
144 & 1,988 & 3,238 & 4,713 \\
192 & 1,265 & 2,524 & 4,117 \\
240 & 0,759 & 1,101 & 2,732 \\
288 & 0,681 & 0,841 & 2,150 \\
336 & 0,558 & 0,703 & 1,810 \\
\hline
\end{tabular}

Observa-se, com base na análise da Tabela 3 e para todas as potências de lâmpadas, num decréscimo da concentração de antocianina com o tempo de exposição à luz. Verifica-se também uma redução acentuada nos cinco primeiros dias de experimento (144 h), com valores de redução do teor de antocianina de $78 \%, 65 \%$ e 47,63\% para as lâmpadas de $10 \mathrm{~W}$, $5 \mathrm{~W}$ e $4 \mathrm{~W}$, respectivamente. Indicando, assim que, dentro do domínio experimental avaliado neste trabalho, os extratos antociânicos mantêm suas características em até $50 \%$ do teor inicial quando submetidos à exposição direta de luz de LED de $4 \mathrm{~W}$ por até 8 dias.

Observa-se também (gráfico 1) que no período de até 240 horas (10 dias), os teores de antocianina apresentam-se com comportamento de decréscimo exponencial, atingindo um assíntota com o tempo da incidência luminosa.

Comportamento semelhante foi obtido por Melo (2001) e Chisté (2008) no estudo de fotodegradação de bixina extraída de sementes de urucum e do extrato antociânico obtido da casca do mangostão, respectivamente.

Gráfico 1 - Teores de antocianinas totais em função do tempo de exposição a diferentes potências de lâmpadas LED.

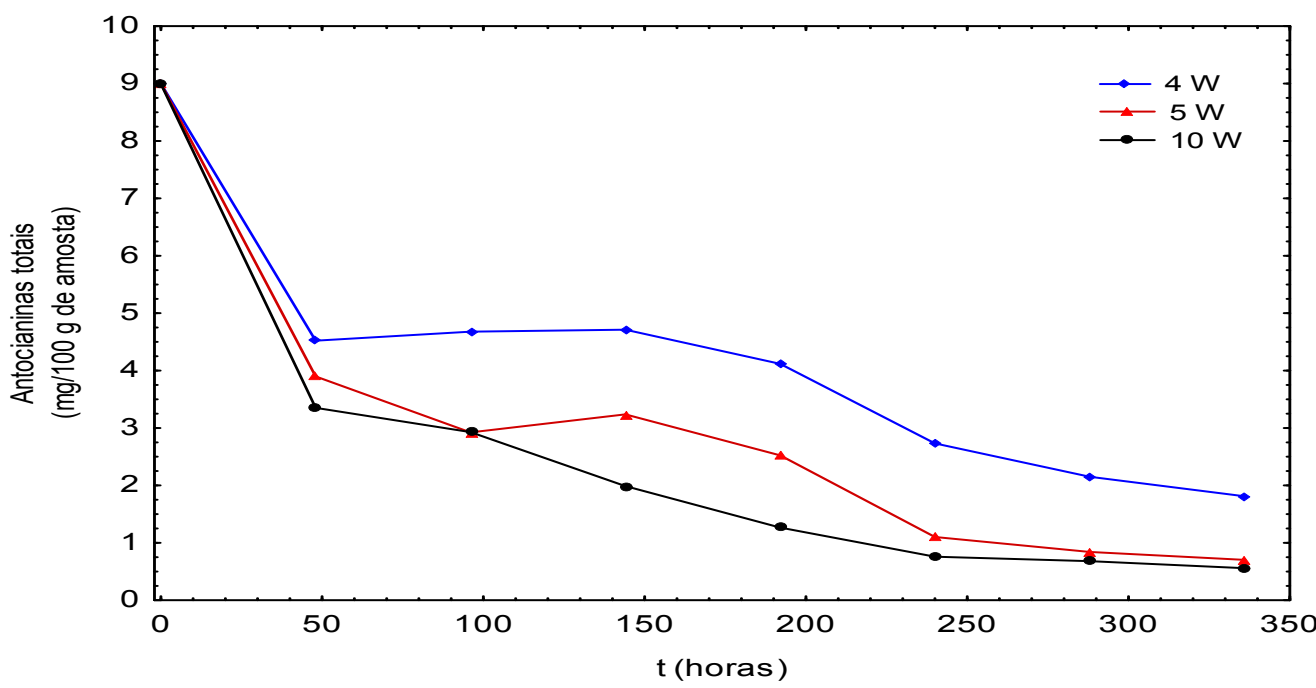




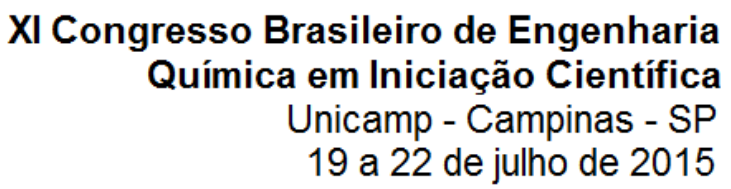

Na Tabela 4 estão representados os parâmetros de ajuste utilizados para avaliar os modelos matemáticos que descrevem a cinética de degradação do pigmento de açaí.

Tabela 4- Parâmetros cinéticos para os extratos antociânicos expostos a incidência direta de luz de LED.

\begin{tabular}{cccccc}
\hline $\begin{array}{c}\text { Potências das } \\
\text { lâmpadas }(\mathrm{W})\end{array}$ & $\begin{array}{c}\text { Ordem da } \\
\text { Reação }\end{array}$ & $\begin{array}{c}\mathrm{C}_{0} \\
(\mathrm{mg} / 100 \mathrm{~g})\end{array}$ & $\begin{array}{c}\mathrm{k} \\
\left(\mathrm{h}^{-1}\right)\end{array}$ & $\mathrm{R}^{2}$ & $\begin{array}{c}\text { Análise de } \\
\text { resíduos }\end{array}$ \\
\hline \multirow{2}{*}{10} & 0 & 5,8843 & 0,0197 & 0,8289 & $\mathrm{~T}$ \\
& 1 & 8,4465 & 0,0119 & 0,9709 & $\mathrm{~T}$ \\
\hline \multirow{2}{*}{5} & 2 & 8,9818 & 0,0033 & 0,9925 & $\mathrm{~A}$ \\
\hline & 1 & 6,3430 & 0,0197 & 0,8626 & $\mathrm{~T}$ \\
& 2 & 8,1273 & 0,0084 & 0,9478 & $\mathrm{~A}$ \\
& 0 & 7,9177 & 0,0023 & 0,9761 & $\mathrm{~A}$ \\
\hline & 1 & 7,8237 & 0,0171 & 0,8859 & $\mathrm{~T}$ \\
& 2 & 8,5215 & 0,0010 & 0,9158 & $\mathrm{~A}$ \\
\hline
\end{tabular}

$\mathrm{C}_{0}=$ Concentração inicial; $\mathrm{k}=$ Constante de velocidade da reação, $\mathrm{R}^{2}=$ coeficiente de determinação; $\mathrm{T}=$ tendencioso; $\mathrm{A}=$ aleatório.

Com base na análise da Tabela 4 observa-se que a fotodegradação do extrato de antocianina do açaí pode ser descrito por uma reação de segunda ordem, uma vez que ambos os parâmetros estatísticos ( $\mathrm{R}^{2}$ e análise de resíduos), utilizados para avaliar a excelência dos modelos, foram satisfatórios, ou seja, o valor do coeficiente de determinação $\left(\mathrm{R}^{2}\right)$, apresenta os maiores valores, quanto à análise de resíduo demonstra total aleatoriedade em todas as condições avaliadas para uma cinética de reação de segunda ordem.

De acordo com os parâmetros cinéticos (constante de velocidade de degradação) obtidos é possível calcular o tempo de meia-vida do extrato antociânico obtido do açaí. O cálculo foi realizado utilizando as equações da Tabela 2 de acordo com o modelo que melhor descreve o processo de fotodegradação dos extratos antociânicos. Na Tabela 5, estão representados os valores obtidos.

Tabela 5. Cálculo do tempo de meia-vida

\begin{tabular}{cc}
\hline Potência de luz de LED $(\mathrm{W})$ & Tempo de meia-vida $\left(\mathrm{t}_{1 / 2}\right)$ (horas) \\
\hline 10 & 33,74 \\
5 & 48,75 \\
4 & 117,35 \\
\hline
\end{tabular}

A análise da tabela 5 demonstra que a amostra submetida à incidência de luz LED a 4W teve maior tempo de meia-vida entre as demais amostras. A amostra perdeu $50 \%$ da concentração inicial depois de 117 horas.

Chisté (2008) verificou que o extrato antociânico de mangostão teve um tempo de meiavida de 597,54 h. Lima, Melo e Lima (2005) estudaram a degradação do extrato antociânico 

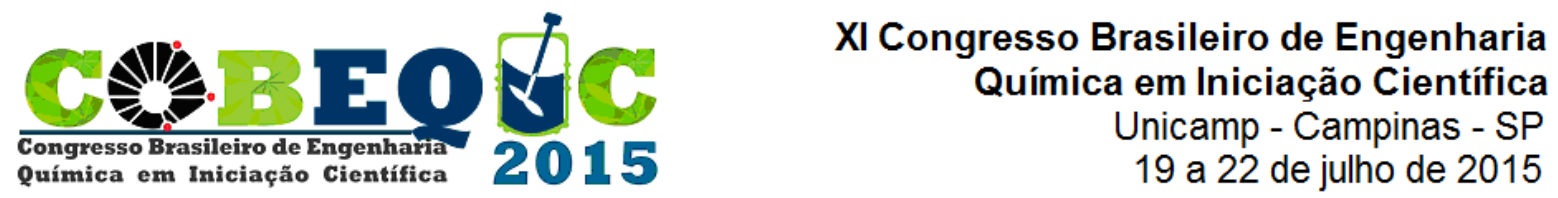

de pitanga roxa frente à luminosidade fluorescente e concluíram que a perda de $50 \%$ da cor original ocorre após $948 \mathrm{~h}$.

\section{CONCLUSÃO}

Através deste trabalho constatou-se um decréscimo significativo dos teores de antocianinas em exposição direta a diferentes potências de lâmpadas de LED no decorrer de tempo. Este decrescimento ocorre de forma mais expressiva nas primeiras horas de exposição, comprovando assim que o extrato antociânico é muito sensível a luz e a exposição direta acelera a degradação. Isso pode ser devido à alta reatividade das antocianinas que degradam rapidamente, além da temperatura média das amostras $\left(30^{\circ} \mathrm{C}\right)$ e da presença de oxigênio.

O efeito da luz LED e da presença de oxigênio na estabilidade de antocianinas de açaí pode ser representado por uma reação de segunda ordem com uma constante de velocidade de degradação na ordem de $1 \times 10^{-3} \mathrm{~h}^{-1}$ e um tempo de meia-vida de 117 horas, o que representa uma redução de $50 \%$ da concentração inicial.

\section{REFERÊNCIAS}

ALEXANDRE, D.; CUNHA, R.; HUBINGER, M.. Conservação do açaí pela tecnologia de obstáculos. Ciência Tecnologia de Alimentos, v. 24, n.1, p. 114-119, 2004.

CHISTÉ, R. C. Estabilidade do Extrato Antociânico Obtido da Casca do Mangostão (Garcinia mangostana L.) 2008. Dissertação de Mestrado em Ciência e Tecnologia de Alimentos da Faculdade de Engenharia de Alimentos, Universidade Federal do Pará, Belém.

CONSTANT, P. B. L. Extração, caracterização e aplicação em antocianinas de açaí. 2003. 199 f. Tese (Doutorado em Ciência e Tecnologia de Alimentos)- UFV, MG, 2003.

FULEKI, T., FRANCIS, F. J.; Quantitative methods for anthocyanins. 1 Extraction and determination of total anthocyanin in craberries. Journal of Food Science. v. 33, p. 72- 77, 1968.

LEES, D. H.; FRANCIS, F. G. Standardization of pigment analysis in cramberries. Hortscienc, [S.I.], v.7, p. 83-84, 1972.

MELO, K. P.; COSTA, C. M. L.; FARIA, L. J. G. Análise da Cinética de fotodegradação de bixina contida em sementes de urucum (Bixa Orellana L.). Revista Brasileira de Corantes Naturais (SBCN), Campina Grande, v. 5, p. 79-85, 2001. 